\title{
Erratum to: Fluorescence control through quantum interference mechanisms in a coupled cavity waveguide
}

\author{
Ronggang Liu' ${ }^{1}$ Tong Liu $^{2}$
}

\section{Erratum to: Opt Quant Electron (2016) 48:186 DOI 10.1007/s11082-016-0463-8}

In the original published article Figs. 1 and 2 were published wrongly (interchanged). This has been corrected in this Erratum.

The authors would like to add a citation in Section 2, "In this section, we consider a four-level atom in the tripod configuration shown in Fig. 1a, where two lower levels...".

Readers must also note that citations of Figs. 1 and 2 throughout the paper are therefore interchanged.

The online version of the original article can be found under doi:10.1007/s11082-016-0463-8.

Ronggang Liu

liurg@hitwh.edu.cn

1 Department of Civil Engineering, Harbin Institute of Technology, Weihai 264209, China

2 Aerospace Research Institute of Materials and Processing Technology, Beijing 10076, China 
Fig. 1 Schematic representation of a four-level atom, which consists of a the upper level $\langle 1|$ is coupled by the same CCW reservoir to the two lower levels $\langle 2|$ and $\langle 3|$, and $\mathbf{b}$ the transition channel between the level $\langle 1|$ and the level $\langle 2|$ is associated with a driving field

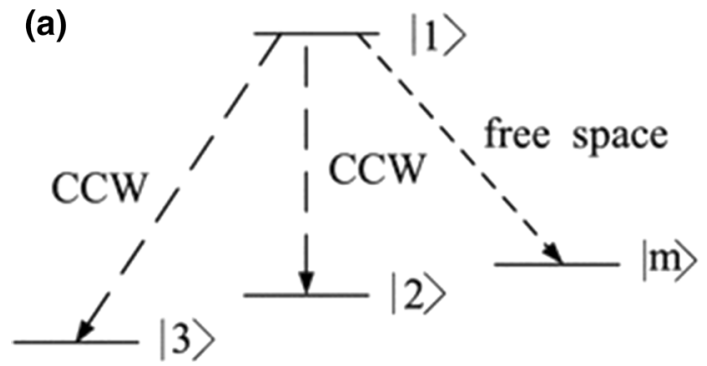

(b)

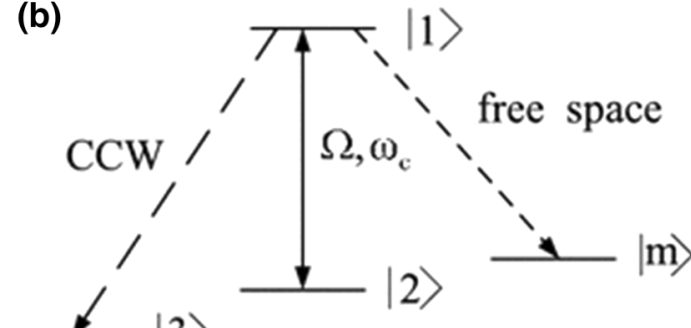

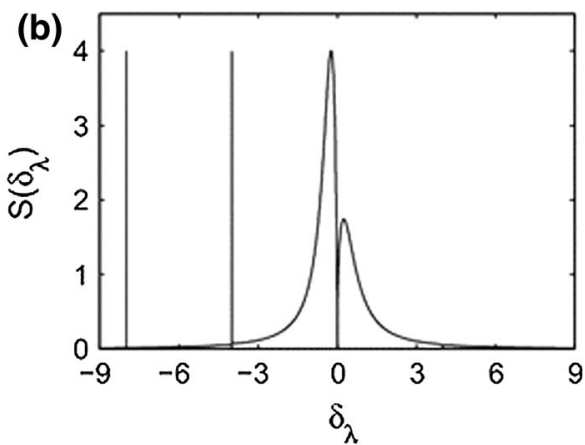
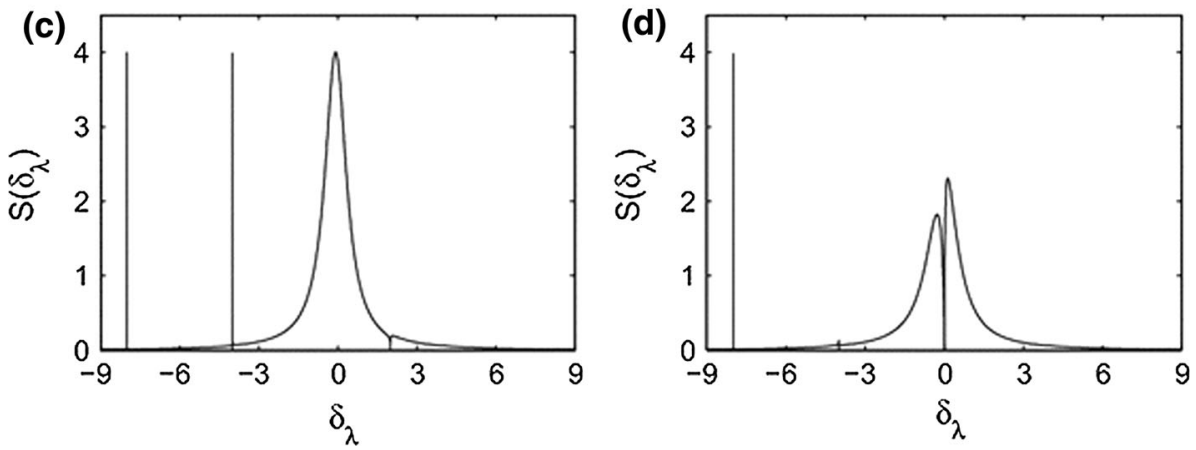

Fig. 2 The spontaneous emission spectra $S\left(\delta_{\lambda}\right)$ for $R=5$ and $W=2$. a $\delta_{12}=-6 ; \delta_{13}=0$; b $\delta_{12}=-6$; $\delta_{13}=2 ; \mathbf{c} \delta_{12}=-6 ; \delta_{13}=4 ; \mathbf{d} \delta_{12}=-6 ; \delta_{13}=-2$ 